\title{
ANOMALOUS TRANSPORT PROPERTIES OF THE QUANTUM LORENTZ GAS
}

\author{
W. HOOGEVEEN and J.A. TJON \\ Institute for Theoretical Physics, Princetonplein 5, P.O. Box 80.006, 3508 TA Utrecht, \\ The Netherlands
}

Received 12 February 1982

\begin{abstract}
The two-scatterer contribution to the Laplace transformed velocity autocorrelation function is investigated with the use of a double contour method which contains the microscopic information in terms of off-shell $T$-matrix elements. By analysing the full off-shell two-scatterer expression both analytically and numerically for a particular model we demonstrate explicitly that this contribution diverges logarithmically for a two-dimensional Lorentz gas, while it is finite for a three-dimensional system. The connection with the classical method of analysis is discussed.

It is shown that the boundstate contributions in three dimensions give rise to an until recently unknown slowly decaying long time tail in the velocity autocorrelation function. Consequently the frequency-dependent electrical conductivity can have a logarithmic singularity at a certain finite frequency.
\end{abstract}

\section{Introduction}

Presently it is a well-established fact that the usual virial expansion of various transport coefficients for classical gases leads to anomalous coefficients ${ }^{1}$ ). It has been shown ${ }^{2}$ ) that resummation of the leading order divergences gives rise to extra terms in the density expansion that depend logarithmically on the density. Since a transport coefficient can be calculated by integrating the appropriate time dependent current correlation function, the slowly decaying long time tails of the velocity autocorrelation function (VACF) as found from molecular dynamics studies ${ }^{3}$ ) and kinetic theory ${ }^{4}$ ) are directly related to the nonanalytic terms in the virial expansions. The same type of nonanalytic behaviour is shown to exist in a classical Lorentz gas ${ }^{5,6}$ ), being a light particle moving in a random distribution of fixed scatterers. Evidence has been presented that also in this system the VACF decays algebraically for long times ${ }^{7}$ ). Unambiguous experimental confirmation of these anomalous phenomena has not been reported yet.

Considerably less is known about quantum systems. Assuming repulsive interactions the nonexistence of the virial expansion has been claimed for both the real quantum gas $^{8}$ ) and the quantum Lorentz gas ${ }^{7}$ ). In both cases quantum mechanical Liouville operators have been used in order to stay close to the classical analysis. From a quantum mechanical point of view the similarities between the descriptions of the two systems are not very obvious. 
In this paper we present a detailed analysis of the one and two scatterer contributions to the density expansion of the Laplace transformed VACF of the quantum Lorentz gas, which is defined in the following way

$$
\hat{S}(\epsilon)=\int_{0}^{\infty} \mathrm{d} t \mathrm{e}^{-\epsilon t}\langle\mathbf{P} \cdot \boldsymbol{P}(t)\rangle \quad(\operatorname{Re} \epsilon \geqslant 0) .
$$

Section 2 contains several definitions and general remarks about the density expansion of $\hat{S}(\epsilon)$. We also introduce in that section a double contour method to express $\hat{S}(\epsilon)$ in terms of off-shell $T$-matrix elements. The method includes in a simple way all statistical correlations. In section 3 we turn to the question of the existence of the virial expansion of the coefficient of self-diffusion $D_{\mathrm{s}}$. This transport coefficient can be studied by considering the small $\epsilon$ behaviour of $\hat{S}(\epsilon)$ since $\left.^{6}\right)$

$$
D_{\mathrm{s}}=\left(m^{2} d\right)^{-1} \operatorname{Re} \lim _{\epsilon \downarrow 0} \hat{S}(\epsilon)
$$

where $d$ is the dimension and $m$ the light particle mass. We assume the interaction to be of a simple separable form so that one and two scatterer $T$-matrix elements can be found explicitly. This enables us to determine the small $\epsilon$ behaviour of the full two-scatterer contribution from the previously derived double contour expressions in a clear and direct manner. Moreover, we have evaluated this contribution numerically over a wide range of $\epsilon$ values. In both cases we find a logarithmic divergence for $\epsilon \downarrow 0$ in two dimensions. For three-dimensional systems the two-scatterer contribution to $D_{s}$ is finite. In section 4 we redefine the problem in terms of Liouville operators in order to make the connection with the standard analyses.

In section 5 we assume the interactions to be attractive. We find that for a three-dimensional system the Fourier transformed VACF $\hat{S}(i \omega)$ has a logarithmic singularity at a certain finite frequency $\omega_{0}$ if the interaction is sufficiently attractive to admit a bound state of the light particle and one scatterer. This singularity occurs because the boundstate contributions to the VACF give rise to a new type of anomalous long time behaviour falling off like $t^{-1} \exp \left(\mathrm{i} \omega_{0} t\right)$ for long times. Consequently the frequency dependent electrical conductivity $\sigma(\omega)$, which is related to $\hat{S}(i \omega)$ in the following way:

$$
\sigma(\omega)=-\left(i e^{2} / \omega m\right)+\left(e^{2} / \hbar \omega m^{2} d\right) \lim _{\eta \downarrow 0}\left[\hat{S}(\epsilon)-\hat{S}^{*}\left(\epsilon^{*}\right)\right] \quad(\epsilon=\eta+i \omega)
$$

exhibits a logarithmic singularity if $\omega \approx \omega_{0}$. A brief account of the study of the boundstate contributions has been given in a recent letter ${ }^{19}$ ). The paper closes with some concluding remarks. 


\section{Density expansion of the velocity autocorrelation function}

The quantum Lorentz gas consists of a single light particle with mass $m$ contained in a $d$-dimensional volume $\Omega_{d}$ that interacts quantum mechanically via a potential $V_{N}$ with a large number $N$ of fixed scatterers at random positions $\boldsymbol{R}^{N} \equiv\left(\boldsymbol{R}_{1}, \ldots, \boldsymbol{R}_{N}\right)$. The Hamiltonian is then given by

$$
H_{N}=H_{0}+V_{N}\left(\boldsymbol{R}^{N}\right)=\boldsymbol{P}^{2}+\sum_{i=1}^{N} v_{i}
$$

We adapt units in which $\hbar=2 m=1, v_{i}$ is the interaction between the light particle and the $i$ th scatterer. In terms of these operators the VACF of the light particle is given by

$$
S(t)=\left\langle\boldsymbol{P} \cdot \boldsymbol{P}^{(N)}(t)\right\rangle=\Omega_{d}^{-N} \int \mathrm{d} \boldsymbol{R}^{N} \operatorname{Tr}\left[\boldsymbol{P} \cdot \boldsymbol{P}^{(N)}(t) \rho_{N}\right]
$$

where

$$
\boldsymbol{P}^{(N)}(t)=\exp \left(\mathrm{i} H_{N} t\right) \boldsymbol{P} \exp \left(-\mathrm{i} H_{N} t\right) .
$$

The density operator is defined in the usual way,

$$
\rho_{N}=Z^{-1} \exp \left(-\beta H_{N}\right) \text {, with } \beta=\left(k_{\mathrm{B}} T\right)^{-1} \text {. }
$$

The averaged partition function $Z$ depends linearly on the volume so that

$$
Z=\left(\Omega_{d} / \Lambda^{d}\right) \zeta(\rho), \quad \text { with } \Lambda=\sqrt{4 \pi \beta},
$$

where $\rho$ is the scatterer density, $\zeta(\rho)$ can be expressed in the familiar cluster expansion").

A density expansion of $S(t)$ is obtained by performing an Ursell expansion for the operators in the right-hand side of eq. (2.2). As a result we find

$$
S(t)=S_{0}+\Lambda^{d} \sum_{n=1}^{\infty}(n !)^{-1} S_{n}(t) \rho^{n}
$$

where $S_{n}(t)$ are the cluster functions, the first three being given by

$$
\begin{aligned}
S_{0}= & \frac{1}{2} d \zeta^{-1} k_{\mathrm{B}} T \\
S_{1}(t)= & \zeta^{-1} \operatorname{Tr}\left[\exp \left(-\beta H_{1}\right) \boldsymbol{P} \cdot \boldsymbol{P}^{(1)}(t)-\exp \left(-\beta H_{0}\right) \boldsymbol{P}^{2}\right] \\
S_{2}(t)= & \zeta^{-1} \int \mathrm{d} \boldsymbol{R} \operatorname{Tr}\left[\exp \left(-\beta H_{2}\right) \boldsymbol{P} \cdot \boldsymbol{P}^{(2)}(t)-2 \exp \left(-\beta H_{1}\right) \boldsymbol{P} \cdot \boldsymbol{P}^{(1)}(t)\right. \\
& \left.+\exp \left(-\beta H_{0}\right) \boldsymbol{P}^{2}\right] .
\end{aligned}
$$

Without loss of generality we assumed $\boldsymbol{R}_{1}=\frac{1}{2} \boldsymbol{R}$ and $\boldsymbol{R}_{2}=-\frac{1}{2} \boldsymbol{R}$. Strictly speaking eq. (2.6) is not yet a density expansion of $S(t)$ since all the cluster 
functions still depend on the density via $\zeta$. The cluster expansion of this function is however known to be well-behaved, so that this part of the statistical density corrections will not give rise to abnormal properties of the correlation function. For this reason we will ignore the density dependence of $\zeta$, so that we take $\zeta=1$.

As in I we will carry out a Watson transformation to obtain the cluster functions in terms of $T$-operators. This procedure consists of two steps. First we transform the time evolution and density operators into a contour integral over the resolvent operators $G_{N}(z) \equiv\left(z-H_{N}\right)^{-1}$,

$$
\begin{aligned}
& \exp \left(\mathrm{i} H_{N} t\right)=(2 \pi \mathrm{i})^{-1} \oint_{C} \mathrm{~d} z_{0} \exp \left(\mathrm{i} z_{0} t\right) G_{N}\left(z_{0}\right) \equiv \mathscr{L}_{0}^{-1} G_{N} \\
& \exp \left[-(\mathrm{it}+\beta) H_{N}\right]=(2 \pi \mathrm{i})^{-1} \oint_{C} \mathrm{~d} z_{\beta} \exp \left[-(\mathrm{it}+\beta) z_{\beta}\right] G_{N}\left(z_{\beta}\right) \equiv \overline{\mathscr{L}}_{\beta}^{-1} G_{N}
\end{aligned}
$$

The contour $C$ has to enclose the cut and all poles of $G_{N}$ in an anti-clockwise direction. Secondly we eliminate the resolvents by substituting

$$
G_{N}=G_{0}+G_{0} T_{N} G_{0} .
$$

Each of the cluster functions can now be considered as being the sum of three terms, hence we find for $S_{1}$ and $S_{2}$

$$
\begin{aligned}
& S_{1}(t)=S_{1}^{\mathrm{r}}(t)+S_{1}^{\mathrm{v}}(t)+S_{1}^{\mathrm{v} 2}(t), \\
& S_{1}^{\mathrm{r}}(t)=\operatorname{Tr}\left[\boldsymbol{P}\left(\mathscr{L}_{0}^{-1} G_{0} T_{1} G_{0}\right) \cdot \mathbf{P}\left(\overline{\mathscr{L}}_{\beta}^{-1} G_{0} T_{1} G_{0}\right)\right], \\
& S_{1}^{\mathrm{v}}(t)=\operatorname{Tr}\left[\mathbf{P}\left(\mathscr{L}_{0}^{-1} G_{0}\right) \cdot \mathbf{P}\left(\overline{\mathscr{L}}_{\beta}^{-1} G_{0} T_{1} G_{0}\right)\right], \\
& S_{1}^{\mathrm{v} 2}(t)=\operatorname{Tr}\left[\mathbf{P}\left(\mathscr{L}_{0}^{-1} G_{0} T_{1} G_{0}\right) \cdot \mathbf{P}\left(\overline{\mathscr{L}}_{\beta}^{-1} G_{0}\right)\right],
\end{aligned}
$$

and

$$
\begin{aligned}
S_{2}(t)= & S_{2}^{\mathrm{r}}(t)+S_{2}^{\mathrm{v} 1}(t)+S_{2}^{\mathrm{v} 2}(t), \\
S_{2}^{\mathrm{r}}(t)= & \int \mathrm{d} \boldsymbol{R} \operatorname{Tr}\left[\boldsymbol{P}\left(\mathscr{L}_{0}^{-1} G_{0} T_{2} G_{0}\right) \cdot \boldsymbol{P}\left(\overline{\mathscr{L}}_{\beta}^{-1} G_{0} T_{2} G_{0}\right)\right. \\
& \left.-2 \mathbf{P}\left(\mathscr{L}_{0}^{-1} G_{0} T_{1} G_{0}\right) \cdot \boldsymbol{P}\left(\overline{\mathscr{L}}_{\beta}^{-1} G_{0} T_{1} G_{0}\right)\right], \\
S_{2}^{\mathrm{v} 1}(t)= & \int \mathrm{d} \boldsymbol{R} \operatorname{Tr}\left[\boldsymbol{P}\left(\mathscr{L}_{0}^{-1} G_{0}\right) \cdot \mathbf{P}\left(\overline{\mathscr{L}}_{\beta}^{-1} G_{0}\left\{T_{2}-2 T_{1}\right\} G_{0}\right)\right], \\
S_{2}^{\mathrm{v} 2}(t)= & \int \mathrm{d} \boldsymbol{R} \operatorname{Tr}\left[\boldsymbol{P}\left(\mathscr{L}_{0}^{-1} G_{0}\left\{T_{2}-2 T_{1}\right\} G_{0}\right) \cdot \mathbf{P}\left(\overline{\mathscr{L}}_{\beta}^{-1} G_{0}\right)\right] .
\end{aligned}
$$

The superscripts $r$ and $v$ stand for real and virtual since the corresponding terms of the cluster functions can be related to the real and virtual collision 
parts of the classical Lorentz-Boltzmann operator, this will be done in section 4. Because of the simple time dependence that follows from eqs. (2.10)-(2.11) the Laplace transformation of each of the cluster functions can readily be done. This yields as factor $\left[\epsilon-\mathrm{i}\left(z_{0}-z_{\beta}\right)\right]^{-1}$ in the integrand, so that we can carry out the $\mathscr{L}_{0}^{-1}$ transformations by closing the $z_{0}$-contour around $z_{\beta}-\mathrm{i} \epsilon$. Clearly the $\overline{\mathscr{L}}_{\beta}^{-1}$ transformations in the v2-terms can be done as well. The resulting expressions for the one and two scatterer contributions are

$$
\begin{aligned}
\hat{S}_{1}^{\mathrm{r}}(\epsilon)= & -(2 \pi)^{-1} \int \mathrm{d} p \mathrm{~d} p^{\prime}\left(\boldsymbol{p} \cdot p^{\prime}\right) \oint \mathrm{d} z \mathrm{e}^{-\beta z} \\
& \times\left[\frac{\left\langle p\left|T_{1}(z-\mathrm{i} \epsilon)\right| p^{\prime}\right\rangle\left\langle p^{\prime}\left|T_{1}(z)\right| p\right\rangle}{\left(z-p^{2}-\mathrm{i} \epsilon\right)\left(z-p^{\prime 2}-\mathrm{i} \epsilon\right)\left(z-p^{\prime 2}\right)\left(z-p^{2}\right)}\right], \\
\hat{S}_{1}^{\mathrm{v} 1}(\epsilon)= & -(2 \pi)^{-1} \int \mathrm{d} p p^{2} \oint \mathrm{d} z \mathrm{e}^{-\beta z}\left[\frac{\left\langle p\left|T_{1}(z)\right| p\right\rangle}{\left(z-p^{2}-\mathrm{i} \epsilon\right)\left(z-p^{2}\right)^{2}}\right], \\
\hat{S}_{1}^{\mathrm{v}}(\epsilon)= & \mathrm{i} \epsilon^{-2} \int \mathrm{d} p \mathrm{e}^{-\beta p^{2}} p^{2}\left\langle p\left|T_{1}\left(p^{2}-\mathrm{i} \epsilon\right)\right| p\right\rangle, \\
\hat{S}_{2}^{\mathrm{r}}(\epsilon)= & -(2 \pi)^{-1} \int \mathrm{d} \boldsymbol{R} \int \mathrm{d} p \mathrm{~d} p^{\prime}\left(p \cdot p^{\prime}\right) \oint \mathrm{d} z \mathrm{e}^{-\beta z} \\
& \times\left[\frac{\left.\left\langle p\left|T_{2}(z-\mathrm{i} \epsilon)\right| p^{\prime}\right\rangle p^{\prime}\left|T_{2}(z)\right| p\right\rangle-2\left\langle p\left|T_{1}(z-\mathrm{i} \epsilon)\right| p^{\prime}\right\rangle\left\langle p^{\prime}\left|T_{1}(z)\right| p\right\rangle}{\left(z-p^{2}-\mathrm{i} \epsilon\right)\left(z-p^{\prime 2}-\mathrm{i} \epsilon\right)\left(z-p^{\prime 2}\right)\left(z-p^{2}\right)}\right], \\
\hat{S}_{2}^{\mathrm{v} 1}(\epsilon)= & -(2 \pi)^{-1} \int \mathrm{d} \boldsymbol{R} \int \mathrm{d} p p^{2} \oint \mathrm{d} z \mathrm{e}^{-\beta 2}\left[\frac{\left\langle p\left|T_{2}(z)\right| p\right\rangle-2\left\langle p\left|T_{1}(z)\right| p\right\rangle}{\left(z-p^{2}-\mathrm{i} \epsilon\right)\left(z-p^{2}\right)^{2}}\right], \\
\hat{S}_{2}^{\mathrm{v} 2}(\epsilon)= & \mathrm{i} \epsilon^{-2} \int \mathrm{d} \boldsymbol{R} \int \mathrm{d} p \mathrm{e}^{-\beta p^{2}} p^{2}\left[\left\langle p\left|T_{2}\left(p^{2}-\mathrm{i} \epsilon\right)\right| p\right\rangle-2\left\langle p\left|T_{1}\left(p^{2}-\mathrm{i} \epsilon\right)\right| p\right\rangle\right] .
\end{aligned}
$$

The contour passes between the singularities in the integrand located at $\operatorname{Im} z=\mathrm{i} \epsilon$ and the real axis (cf. fig. 1).

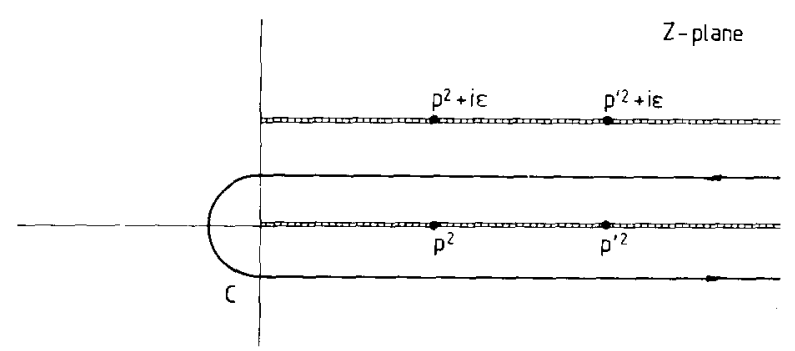

Fig. 1. Singularity structure of the real collision contribution for the case of repulsive interactions. 
The density expansion for $\hat{S}(\epsilon)$ that follows from eq. (2.6) cannot simply be used to study the diffusion coefficient eq. (1.2), since for $\epsilon \rightarrow 0, S_{n}(\epsilon)$ becomes $\mathcal{O}\left(\epsilon^{-(n+1)}\right)$. These leading divergences however progress geometrically and can be removed explicitly following Zwanzig's resummation method ${ }^{12}$ ). If the density expansion for $\hat{S}(\epsilon)$ has the form

$$
\hat{S}(\epsilon)=(\Lambda / 2 \pi)^{d} \int \mathrm{d} p \mathrm{e}^{-\beta p^{2}} p^{2}\left[\epsilon^{-1}+\sum_{n=1}^{\infty} s_{n}(\epsilon) \rho^{n}\right],
$$

new functions $c_{n}(\epsilon)$ can be defined by inverting the integrand

$$
\hat{S}(\epsilon)=(\Lambda / 2 \pi)^{d} \int \mathrm{d} p \mathrm{e}^{-\beta p^{2}} p^{2}\left[\epsilon+\sum_{n=1}^{\infty} c_{n}(\epsilon) \rho^{n}\right]^{-1},
$$

so that

$$
\begin{aligned}
& c_{1}=-\epsilon^{2} s_{1}, \\
& c_{2}=-\epsilon^{2} s_{2}+\epsilon^{3} s_{1}^{2} .
\end{aligned}
$$

As a result the leading order divergences are absent in $c_{n}$. For two-dimensional classical systems $c_{2}$ is still logarithmically divergent for $\epsilon \rightarrow 0$. In the next section we will study $\hat{S}_{1}$ and $\hat{S}_{2}$ in detail to find out whether this is also the case in the quantum mechanical situation.

\section{Small $\epsilon$ behaviour of $\hat{S}_{1}$ and $\hat{S}_{2}$}

Following I we choose the interaction to be of a separable form, as a result of this the multiple integral expressions for the cluster functions factorize and can be analysed in detail. Given the interaction to be of the following form,

$$
\left\langle\boldsymbol{p}|v| \boldsymbol{p}^{\prime}\right\rangle=\lambda g(p) g\left(p^{\prime}\right),
$$

the one and two scatterer $T$-matrix elements are readily found to be given by $^{11}$ )

$$
\begin{aligned}
& \left\langle p\left|T_{1}(z)\right| p^{\prime}\right\rangle=\lambda g(p) g\left(p^{\prime}\right) \Delta^{-1}(z), \\
& \left\langle p\left|T_{2}(z)\right| p^{\prime}\right\rangle=2 \lambda g(p) g\left(p^{\prime}\right)\left[\frac{\Delta(z) \cos \frac{1}{2} R \cdot\left(p-p^{\prime}\right)+D(R ; z) \cos \frac{1}{2} R \cdot\left(p+p^{\prime}\right)}{\Delta^{2}(z)-D^{2}(R ; z)}\right],
\end{aligned}
$$

where

$$
\begin{aligned}
& \Delta(z)=1-\lambda \int \mathrm{dpg} g^{2}(p)\left(z-p^{2}\right)^{-1}, \\
& D(R ; z)=\lambda \int \mathrm{dp} \exp (\mathrm{i} R \cdot p) g^{2}(p)\left(z-p^{2}\right)^{-1} .
\end{aligned}
$$


The final analysis of $\hat{S}_{2}$ is facilitated one further step by choosing a particular form for the interaction. This is not a severe restraint since the characteristic small $\epsilon$-behaviour of the cluster functions is believed to be independent of the detailed shape of the interaction. The $S$-wave effective range potential (i.e. $\left.g(p) \equiv\left(p^{2}+\alpha^{2}\right)^{1 / 2}\right)$ turns out to be a convenient choice, because for both two and three dimensions the denominator functions can be expressed in terms of simple standard functions; the explicit expressions are stated in appendix A.

For an isotropic interaction the real one-scatterer contribution (2.21) vanishes. It is obvious that the leading order terms of the virtual one-scatterer contributions are complex conjugates of each other, hence

$$
\hat{S}_{1}(\epsilon)=2 \epsilon^{-2} \int \mathrm{d} p \mathrm{e}^{-\beta p^{2}} p^{2} \operatorname{Im}\left\langle p\left|T_{1}\left(p^{2}+\mathrm{i} 0\right)\right| p\right\rangle+\mathcal{O}\left(\epsilon^{-1}\right) .
$$

Since $T_{1}$ satisfies the unitarity condition

$$
T_{1}-T_{1}^{\dagger}=T_{1}\left(G_{0}-G_{0}^{\dagger}\right) T_{1}^{\dagger},
$$

we see that eq. (3.6) is just the classical Boltzmann result with a quantummechanical cross-section. If the interaction should admit boundstates this would not change the leading order terms of $\hat{S}_{1}(\epsilon)$. We will use the separability assumption explicitly to study $\hat{S}_{2}(\epsilon)$. Let us first consider $\hat{S}_{2}^{\text {v2 }}(\epsilon)$, eq. (2.26), for a two-dimensional system. From the explicit form of the $T_{2}$-matrix elements (3.3) it is clear that we can eliminate all angular integrals, so that we find

$$
\begin{aligned}
\hat{S}_{2}^{\mathrm{v} 2}(\epsilon)= & 4 \mathrm{i} \pi^{2} \lambda \epsilon^{-2} \int_{0}^{\infty} \mathrm{d} R R \int_{0}^{\infty} \mathrm{d} E \mathrm{e}^{-\beta E} E g^{2}(p) \\
& \times\left[\frac{\Delta(E-\mathrm{i} \epsilon)+J_{0}(R p) D(R ; E-\mathrm{i} \epsilon)}{\Delta^{2}(E-\mathrm{i} \epsilon)-D^{2}(R ; E-\mathrm{i} \epsilon)}-\frac{1}{\Delta(E-\mathrm{i} \epsilon)}\right],
\end{aligned}
$$

where $J_{0}$ is a zeroth order Bessel function and $p=\sqrt{E}$. On physical grounds it is clear that the small $\epsilon$ (i.e. long time) behaviour of the cluster functions will be determined mainly by those collision sequences where all the participating scatterers are far apart. The obvious means to study the large $R$ behaviour of the integrand of $\hat{S}_{2}^{\mathrm{v2}}$ is the multiple scattering series of $T_{2}$. This series is equivalent to the series we obtain after expanding the integrand of eq. (3.8) into powers of $D$, the expression between square brackets then reads as follows:

$$
\Delta^{-1} \sum_{n_{-}=1}^{\infty}(D / \Delta)^{2 n_{-}}+\Delta^{-1} J_{0} \sum_{n_{+}=0}^{\infty}(D / \Delta)^{2 n_{+}+1}
$$

Each term of the first sum corresponds to an odd number of scatterings of the 
light particle, starting with three collisions. The even collision sequences are summed in the second term. In appendix $A$ we show that for the effective range interaction the two-dimensional $D$-function has the following asymptotic form for large $R$ :

$$
D(R ; E-\mathrm{i} \epsilon)=-\pi \lambda g^{2}(p) \sqrt{\frac{2 \pi}{\mathrm{i} R p}} \exp (-R \sqrt{-E+\mathrm{i} \epsilon})\left[1+\frac{\mathrm{i}}{8 R p}+\mathcal{O}\left(R^{-2}\right)\right] .
$$

We also need the asymptotic form of the Bessel function ${ }^{13}$ ),

$$
\begin{aligned}
J_{0}(R p)= & \sqrt{\frac{2}{\pi R p}}\left[\left(1+\mathcal{O}\left(R^{-2}\right)\right) \cos \left(R p-\frac{\pi}{4}\right)\right. \\
& \left.+\left(\frac{1}{8 R p}+\mathcal{O}\left(R^{-3}\right)\right) \sin \left(R p-\frac{\pi}{4}\right)\right] .
\end{aligned}
$$

If we combine these two asymptotic expansions with eqs. (3.8)-(3.9) we get the following expression for $\hat{S}_{2}^{\mathrm{v} 2}$

$$
\begin{aligned}
\hat{S}_{2}^{\mathrm{v}}(\epsilon)= & 4 \pi^{3} \lambda^{2} \epsilon^{-2} \int \mathrm{d} R \int \mathrm{d} E \mathrm{e}^{-\beta E} p\left[2 \pi^{2} \lambda g^{6}(p) \Delta^{-3}(E-\mathrm{i} \epsilon)\right. \\
& \times \exp (-2 \sqrt{-E+\mathrm{i} \epsilon})\left\{1+\frac{\mathrm{i}}{4 R p}+\mathcal{O}\left(R^{-2}\right)\right\} \\
& -g^{4}(p) \Delta^{-2}(E-\mathrm{i} \epsilon)\left(\exp [R(\mathrm{i} p-\sqrt{-E+\mathrm{i} \epsilon})]\left\{1+\mathcal{O}\left(R^{-2}\right)\right\}\right. \\
& \left.\left.+\mathrm{i} \exp [-R(\mathrm{i} p+\sqrt{-E+\mathrm{i} \epsilon})]\left\{1+\frac{\mathrm{i}}{4 R p}+\mathcal{O}\left(R^{-2}\right)\right\}\right)\right] .
\end{aligned}
$$

The first term originates from the odd collision term and yields a finite contribution to $\epsilon^{2} \hat{S}_{2}^{v 2}(\epsilon)$. The second term in eq. (3.11) is evidently $\mathcal{O}\left(\epsilon^{-3}\right)$, we note that the $O\left(R^{-1}\right)$ contributions to this term cancel each other so there is no $\log \epsilon$ term here. As a result we find

$$
\hat{S}_{2}^{v 2}(\epsilon)=-8 \pi^{3} \epsilon^{-3} \int_{0}^{\infty} \mathrm{d} E \mathrm{e}^{-\beta E} E\left\langle p\left|T_{1}(E-\mathrm{i} 0)\right| p\right\rangle^{2}+\mathcal{O}\left(\epsilon^{-2}\right) .
$$

The leading order term will be removed by the Zwanzig resummation, the remainder yields a finite contribution to the virial expansion. The first virtual collision term (2.25) has the same kind of asymptotic behaviour; as for the one-scatterer situation the leading order terms of $\hat{S}_{2}^{\mathrm{v} 1}$ and $\hat{S}_{2}^{\mathrm{v} 2}$ are complex conjugates of each other. The extra contour integral does not pose a serious problem which is shown for the case of $\hat{S}_{2}^{r}$ below. In a three-dimensional system the asymptotic behaviour of the virtual term is essentially the same, some details can be found in appendix A.

We now turn to the real two-scatterer collisions. With the use of the 
separable $T$-matrix expression, eqs. (3.2)-(3.3), we see that the integrals over the momenta in eq. (2.24) can be factorized yielding

$$
\begin{aligned}
\hat{S}_{2}^{\mathrm{r}}(\epsilon)= & 2 \pi^{-1} \lambda^{2} \epsilon^{-2} \int \mathrm{d} R \oint \mathrm{d} z \mathrm{e}^{-\beta z} \tau(R ; z, \epsilon) \\
& \times\left[\frac{\Delta(z-\mathrm{i} \epsilon) \Delta(z)-D(R ; z-\mathrm{i} \epsilon) D(R ; z)}{\left(\Delta^{2}(z-\mathrm{i} \epsilon)-D^{2}(R ; z-\mathrm{i} \epsilon)\right)\left(\Delta^{2}(z)-D^{2}(R ; z)\right)}\right],
\end{aligned}
$$

where

$$
\tau(R ; z, \epsilon)=\epsilon^{2} \int \mathrm{d} p \mathrm{~d} p^{\prime}\left[\frac{g^{2}(p) g^{2}\left(p^{\prime}\right)\left(p \cdot p^{\prime}\right) \cos ^{2} \frac{1}{2} R \cdot\left(p+p^{\prime}\right)}{\left(z-p^{2}-\mathrm{i} \epsilon\right)\left(z-p^{2}\right)\left(z-p^{\prime 2}-\mathrm{i} \epsilon\right)\left(z-p^{\prime 2}\right)}\right] .
$$

In the limit of small $\epsilon$ we only have to consider the upper half of the $z$-contour since

$$
\lim _{\epsilon \downarrow 0} \tau(R ; z, \epsilon)=0, \quad \text { if } \operatorname{Im} z<0,
$$

so that we can take in eq. (3.14) $z=E+\frac{1}{2} \mathrm{i} \epsilon$ and integrate over all positive values of $E$. Expanding the expression between square brackets in eq. (3.14) into powers of $D$ we find

$$
|\Delta|^{-2}\left[\sum_{n_{-}=0}^{\infty} \sum_{m_{-}=0}^{\infty}\left(D_{-} / \Delta_{-}\right)^{2 n_{-}}\left(D_{+} / \Delta_{+}\right)^{2 m_{-}}-\sum_{n_{+}=0}^{\infty} \sum_{m_{+}=0}^{\infty}\left(D_{-} / \Delta_{-}\right)^{2 n_{+}+1}\left(D_{+} / \Delta_{+}\right)^{2 m_{+}+1}\right],
$$

with $D_{ \pm}=D\left(E \pm \frac{1}{2} \mathrm{i} \epsilon\right)$ and $\Delta_{ \pm}=\Delta\left(E \pm \frac{1}{2} \mathrm{i} \epsilon\right)$. The other terms with an even and an odd power of $D$ give on symmetry grounds a vanishing contribution to eq. (3.14), and as a result have been dropped in eq. (3.17). For the two-dimensional effective range model eq. (3.15) has the following asymptotic form for large $R$ (see appendix $\mathrm{A}$ ):

$$
\begin{aligned}
\tau\left(R ; E+\frac{1}{2} \mathrm{i} \epsilon, \epsilon\right)= & -(R p)^{-1} \pi^{3} E g^{4}(p) \\
& \times\left[-\mathrm{i} \exp \left(-2 R \sqrt{-E+\frac{1}{2} \mathrm{i} \epsilon}\right)\left(1-\frac{3 \mathrm{i}}{4 R p}+\mathcal{O}\left(R^{-2}\right)\right)\right. \\
& +\mathrm{i} \exp \left(-2 \sqrt{-E-\frac{1}{2} \mathrm{i} \epsilon}\right)\left(1+\frac{3 \mathrm{i}}{4 R p}+\mathcal{O}\left(R^{-2}\right)\right) \\
& \left.+2 \exp \left[-R\left(\sqrt{-E+\frac{1}{2} \mathrm{i} \epsilon}+\sqrt{-E-\frac{1}{2} \mathrm{i} \epsilon}\right)\right]\left(1+\mathcal{O}\left(R^{-2}\right)\right)\right],
\end{aligned}
$$

with $p=\sqrt{E}$. Substituting eqs. (3.10), (3.18) and (3.17) in eq. (3.14) we find for small $\epsilon$ 


$$
\begin{aligned}
\hat{S}_{2}^{\mathrm{r}}(\epsilon)= & 8 \pi^{3} \lambda^{2} \epsilon^{-2} \int \mathrm{d} R \int \mathrm{d} E \mathrm{e}^{-\beta E} p g^{4}(p)|\Delta(E+\mathrm{i} 0)|^{-2} \\
& \times \exp \left[-R\left(\sqrt{-E+\frac{1}{2} \mathrm{i} \epsilon}+\sqrt{-E-\frac{1}{2} \mathrm{i} \epsilon}\right)\right] \\
& -32 \pi^{6} \lambda^{4} \epsilon^{-2} \int \mathrm{d} R \int \mathrm{d} E \mathrm{e}^{-\beta E} g^{8}(p) R^{-1} \\
& \times \exp \left[-2 R\left(\sqrt{-E+\frac{1}{2} \mathrm{i} \epsilon}+\sqrt{-E-\frac{1}{2} \mathrm{i} \epsilon}\right)\right] \\
& \times|\Delta(E+\mathrm{i} 0)|^{-2}\left(\operatorname{Im} \Delta^{-1}(E+\mathrm{i} 0)\right)^{2}+O\left(\epsilon^{-2}\right)
\end{aligned}
$$

All terms containing exponentials with non vanishing arguments for $\epsilon \rightarrow 0$ lead to $O\left(\epsilon^{-2}\right)$ contributions. Using the Zwanzig resummation the first term leads to a finite contribution to the diffusion coefficient, while the second term yields a logarithmically divergent contribution. We may obtain an explicit asymptotic expression for eq. (3.19) by evaluating the $R$-integral. In order to get the final result in terms of scattering cross-sections we also apply the optical theorem which is for our isotropic model simply given by

$$
\operatorname{Im}\left\langle p\left|T_{1}(E+i 0)\right| p\right\rangle=-\pi^{2}\left|\left\langle p\left|T_{1}(E+i 0)\right| p\right\rangle\right|^{2} .
$$

So

$$
\begin{aligned}
\hat{S}_{2}^{\mathrm{r}}(\epsilon)= & 16 \pi^{3} \epsilon^{-3} \int_{0}^{\infty} \mathrm{d} E \mathrm{e}^{-\beta E} E\left|\left\langle p\left|T_{1}(E+\mathrm{i} 0)\right| p\right\rangle\right|^{2} \\
& +32 \pi^{10} \epsilon^{-2} \log (\epsilon) \int_{0}^{\infty} \mathrm{d} E \mathrm{e}^{-\beta E}\left|\left\langle p\left|T_{1}(E+\mathrm{i} 0)\right| p\right\rangle\right|^{6}+\mathcal{O}\left(\epsilon^{-2}\right) .
\end{aligned}
$$

In appendix $A$ we show that for a three-dimensional system there is no $\log \epsilon$ divergence here. Combination of all real and virtual one and two scatterer contributions yields the following for the Laplace transfromed VACF (2.27) in two dimensions:

$$
\begin{aligned}
\hat{S}(\epsilon)= & (4 \pi)^{-1} \Lambda^{2} \int_{0}^{\infty} \mathrm{d} E \mathrm{e}^{-\beta E} E\left[\epsilon^{-1}-\rho\left\{8 \pi^{4} \epsilon^{-2}\left|\left\langle p\left|T_{1}(E+\mathrm{i} 0)\right| p\right\rangle\right|^{2}+\mathcal{O}\left(\epsilon^{-1}\right)\right\}\right. \\
& +\rho^{2}\left\{64 \pi^{8} \epsilon^{-3}\left|\left\langle p\left|T_{1}(E+\mathrm{i} 0)\right| p\right\rangle\right|^{4}+64 \pi^{11} E^{-1} \epsilon^{-2} \log (\epsilon)\left|\left\langle p\left|T_{1}(E+\mathrm{i} 0)\right| p\right\rangle\right|^{6}\right. \\
& \left.\left.+O\left(\epsilon^{-2}\right)\right\}+O\left(\rho^{3} \epsilon^{-4}\right)\right]
\end{aligned}
$$

We have also analysed the two-dimensional cluster functions numerically. The trace integrals in eqs. (2.24)-(2.25) can be done analytically for the effective range model, so in order to evaluate the real and virtual terms a double integral remains.

To attain numerical stability in the large $R$ region a double subtraction was needed. The results of this calculation are shown in fig. 2 , where the solid 


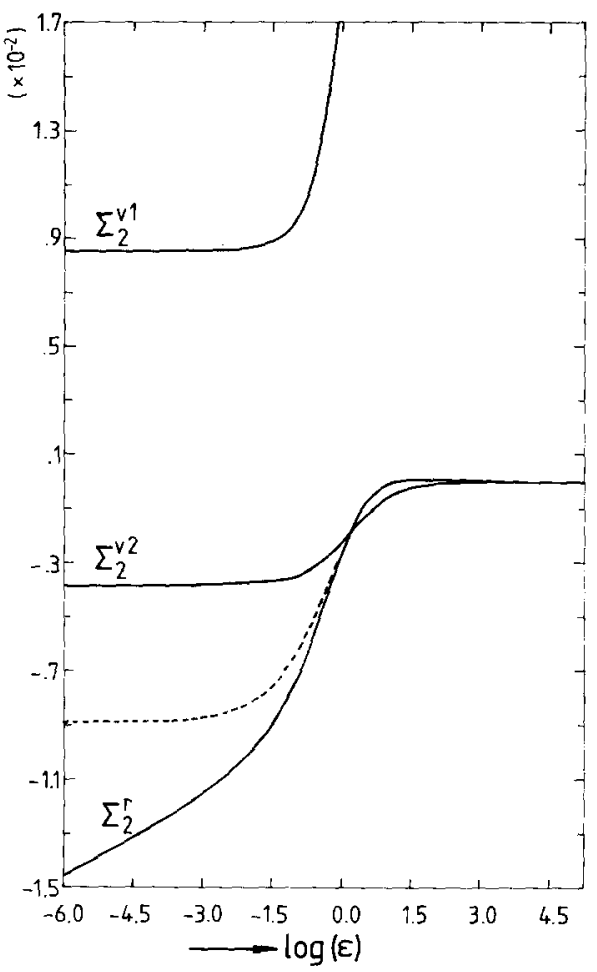

Fig. 2. Two-dimensional real and virtual two-scatterer collision contributions for a repulsive potential $\left(l \equiv 2 \pi^{2} \alpha^{-1} \lambda=1, \alpha=1\right)$ at a temperature $\beta=1$. The dashed line (-- ) represents $\Sigma_{2}^{r}$ minus the corresponding logarithmic term that follows from eq. (3.21).

lines represent the following functions:

$$
\begin{aligned}
& \Sigma_{2}^{\mathrm{r}}(\epsilon)=\epsilon^{2} \operatorname{Re}\left[\hat{S}_{2}^{\mathrm{r}}(\epsilon)-16 \pi^{3} \epsilon^{-3} \int_{0}^{\infty} \mathrm{d} E \mathrm{e}^{-\beta E} E\left|\left\langle p\left|T_{1}(E+\mathrm{i} 0)\right| p\right\rangle\right|^{2}\right], \\
& \Sigma_{2}^{\mathrm{v} 1}(\epsilon)=\epsilon^{2} \operatorname{Re}\left[\hat{S}_{2}^{\mathrm{v} \mathrm{t}}(\epsilon)+8 \pi^{3} \epsilon^{-3} \int_{0}^{\infty} \mathrm{d} E \mathrm{e}^{-\beta E} E\left\langle p\left|T_{1}(E-\mathrm{i} 0)\right| p\right\rangle^{2}\right], \\
& \Sigma_{2}^{\mathrm{v} 2}(\epsilon)=\epsilon^{2} \operatorname{Re}\left[\hat{S}_{2}^{\mathrm{v} 2}(\epsilon)+8 \pi^{3} \epsilon^{-3} \int_{0}^{\infty} \mathrm{d} E \mathrm{e}^{-\beta E} E\left\langle p\left|T_{1}(E+\mathrm{i} 0)\right| p\right\rangle^{2}\right] .
\end{aligned}
$$

The numerical values found for these functions for small $\epsilon$ are all in complete agreement with the analytic estimates made in this section. In particular, the difference between $\Sigma_{2}^{r}$ and the estimate of the logarithmic term of $\hat{S}_{2}^{r}$ should go to a finite value for $\epsilon \rightarrow 0$. This result is shown in fig. 2 (dashed line). 


\section{Relation with the classical approach}

For a classical system the time evolution of the momenta is usually described by a Liouville operator. In order to benefit from the classical results the time evolution in a quantum system is most often described by a Liouville operator as well. The disadvantage of this method is the fact that one has to manipulate with several unfamiliar tetradic operators. For this reason we adapted the double contour method, where we could express the correlation function directly in terms of well known $T$-operators. In this section we relate the two methods.

Let us start by mentioning some definitions and general properties of the Liouville and related operators. Using a Liouville operator $\mathscr{L}_{N}$ to describe the time evolution we have to rewrite eq. (2.3) to

$$
\boldsymbol{P}^{(N)}(t)=\exp \left(\mathrm{i} \mathscr{L}_{N} t\right) \boldsymbol{P} .
$$

$\mathscr{L}_{N}$ is a tetradic operator with the following multiplication rule,

$$
\left\langle p\left|\mathscr{L}_{N} A\right| \mathbf{q}\right\rangle=\int \mathrm{d} p^{\prime} \mathrm{d} q^{\prime}\left(\mathbf{p}, \mathbf{q}\left|\mathscr{L}_{N}\right| \mathbf{p}^{\prime}, \mathbf{q}^{\prime}\right)\left\langle\mathbf{p}^{\prime}|A| \mathbf{q}^{\prime}\right\rangle
$$

where $A$ is some ordinary operator. The tetradic matrix elements are simply related to the Hamiltonian by introducing the tetradic version of $H_{N}$ and its transposed

$$
\begin{aligned}
& \left(\boldsymbol{p}, \boldsymbol{q}\left|\mathscr{H}_{N}\right| \mathbf{p}^{\prime}, \boldsymbol{q}^{\prime}\right)=\left\langle\boldsymbol{p}\left|H_{N}\right| \boldsymbol{p}^{\prime}\right\rangle \delta\left(\boldsymbol{q}-\boldsymbol{q}^{\prime}\right), \\
& \left(\boldsymbol{p}, \boldsymbol{q}\left|\tilde{\mathscr{H}}_{N}\right| \boldsymbol{p}^{\prime}, \boldsymbol{q}^{\prime}\right)=\delta\left(\boldsymbol{p}-\boldsymbol{p}^{\prime}\right)\left\langle\boldsymbol{q}^{\prime}\left|H_{N}\right| \boldsymbol{q}\right\rangle,
\end{aligned}
$$

so that

$$
\mathscr{L}_{\mathrm{N}}=\mathscr{H}_{\mathrm{N}}-\tilde{\mathscr{H}}_{\mathrm{N}}
$$

Related to $\mathscr{L}_{\mathrm{N}}$ are its Greens function $\mathscr{G}_{\mathrm{N}}$

$$
\mathscr{G}_{N}(\chi)=\left(\chi-\mathscr{L}_{N}\right)^{-1}
$$

and a tetradic collision operator $\mathscr{W}_{N}$ which is defined by the following relation:

$$
\mathscr{G}_{N}(\chi)=\mathscr{G}_{0}(\chi)+\mathscr{G}_{0}(\chi) \mathscr{W}_{N}(\chi) \mathscr{G}_{0}(\chi)
$$

The relation between this tetradic collision operator and the usual (dyadic) $T_{N}$ is given by Fano's formula ${ }^{14}$ )

$$
\begin{aligned}
\mathscr{W}_{N}(\chi)= & \mathscr{T}_{N}\left(\tilde{\mathscr{H}}_{0}+\chi\right)-\tilde{\mathscr{T}}_{N}\left(\mathscr{H}_{0}-\chi\right)+(2 \pi \mathrm{i})^{-1} \oint_{C} \mathrm{~d} z\left[\mathscr{R}_{0}(z+\chi)-\tilde{\mathscr{R}}_{0}(z)\right] \\
& \times \mathscr{T}_{N}(z+\chi) \tilde{\mathscr{T}}_{N}(z)\left[\mathscr{R}_{0}(z+\chi)-\tilde{\mathscr{R}}_{0}(z)\right],
\end{aligned}
$$


$\mathscr{T}_{N}$ and $\mathscr{R}_{0}$ are the tetradic versions of $T_{N}$ and $G_{0}$ respectively, having the following matrix elements

$$
\begin{aligned}
& \left(\boldsymbol{p}, \boldsymbol{q}\left|\mathscr{T}_{N}(z)\right| \boldsymbol{p}^{\prime}, \boldsymbol{q}^{\prime}\right)=\left\langle\boldsymbol{p}\left|T_{N}(z)\right| \boldsymbol{p}^{\prime}\right\rangle \delta\left(\boldsymbol{q}-\boldsymbol{q}^{\prime}\right), \\
& \left(\boldsymbol{p}, \boldsymbol{q}\left|\mathscr{R}_{0}(z)\right| \boldsymbol{p}^{\prime}, \boldsymbol{q}^{\prime}\right)=\left\langle\boldsymbol{p}\left|G_{0}(z)\right| \boldsymbol{p}^{\prime}\right\rangle \delta\left(\boldsymbol{q}-\boldsymbol{q}^{\prime}\right) .
\end{aligned}
$$

$C$ is again the Watson contour that we used earlier in section 2. In terms of these operators we find for the Laplace transformed VACF

$$
\hat{S}(\epsilon)=\epsilon^{-1}\left\langle\mathbf{P}^{2}\right\rangle-\mathrm{i}\left\langle\mathbf{P} \mathscr{G}_{0}(\chi)^{\mathscr{W}} \mathcal{W}_{N}(\chi)^{\mathscr{G}} \mathscr{G}_{0}(\chi) \mathbf{P}\right\rangle,
$$

$\chi=-\mathrm{i} \epsilon$ from now on. The usual way to obtain a density expansion of $\hat{S}(\epsilon)$ analogous to eq. (2.6) is by making a cluster expansion of $\mathscr{W}_{N}$ replacing $\rho_{N}$ by $\rho_{0}$. Then we find the following for the one scatterer cluster function, eq. (2.8):

$$
\hat{S}_{1}(\epsilon)=\mathbf{i} \epsilon^{-2} \int \mathrm{d} p \mathrm{~d} \boldsymbol{p}^{\prime} \mathrm{e}^{-\beta p^{2}}\left(\boldsymbol{p} \cdot \boldsymbol{p}^{\prime}\right)\left(\boldsymbol{p}, \boldsymbol{p}\left|\mathscr{W}_{1}(\chi)\right| \boldsymbol{p}^{\prime}, \boldsymbol{p}^{\prime}\right) .
$$

With the use of Fano's formula for $\mathscr{W}_{1}$ one readily recognises the virtual and the real collision terms of $\hat{S}_{1}$, as defined in eqs. (2.21)-(2.23). Obviously approximating $\rho_{N}$ by $\rho_{0}$ amounts to replacing $\mathrm{e}^{-\beta z}$ by $\mathrm{e}^{-\beta E}$ in these expressions. We note that only the "diagonal" matrix elements of $\mathscr{W}_{1}$ appear. If we apply Fano's formula to them for $\epsilon \downarrow 0$ and subsequently the unitarity relation for the $T$-operators (3.7), we find that $\left(p, p\left|W_{1}\right| p^{\prime}, p^{\prime}\right)$ tends to the classical Lorentz-Boltzmann operator ${ }^{5}$ ) with a quantum-mechanical cross-section

$$
\begin{aligned}
\lim _{\epsilon \downarrow 0}\left(p, p\left|W_{1}(\chi)\right| p^{\prime}, p^{\prime}\right) & =-2 \pi \mathrm{i}\left[\delta\left(p^{2}-p^{\prime 2}\right)\left|\left\langle p\left|T_{1}\left(p^{2}+\mathrm{i} 0\right)\right| p^{\prime}\right\rangle\right|^{2}\right. \\
& \left.-\delta\left(p-p^{\prime}\right) \int \mathrm{d} p^{\prime \prime}\left|\left\langle p\left|T_{1}\left(p^{2}+\mathrm{i} 0\right)\right| p^{\prime \prime}\right\rangle\right|^{2} \delta\left(p^{\prime \prime 2}-p^{2}\right)\right] .
\end{aligned}
$$

This justifies the nomenclature we introduced in section 2 .

Analogous to $\hat{S}_{1}$, eq. (4.12), we find for $\hat{S}_{2}(\epsilon)$

$$
\hat{S}_{2}(\epsilon)=\mathrm{i} \epsilon^{-2} \int \mathrm{d} \boldsymbol{R} \int \mathrm{d} p \mathrm{~d} p^{\prime} \mathrm{e}^{-\beta p^{2}}\left(p \cdot p^{\prime}\right)\left(p, p\left|W_{2}(\chi)-2 W_{1}(\chi)\right| p^{\prime}, p^{\prime}\right) .
$$

As in the classical case we can make a binary collision expansion of $\mathscr{W}_{2}$. The relation between the matrix elements of $\mathscr{W}_{1}\left(\chi ; \boldsymbol{R}_{1}\right)$ describing scattering from a centre at $\boldsymbol{R}_{1}$, and $\mathcal{W}_{1}(\chi)$ is found to be

$$
\begin{aligned}
\left(\boldsymbol{p}, \boldsymbol{q}\left|\mathscr{W}_{1}\left(\chi ; \boldsymbol{R}_{1}\right)\right| \boldsymbol{p}^{\prime}, \boldsymbol{q}^{\prime}\right)= & \exp \left[\mathrm{i} \boldsymbol{R}_{1} \cdot(\boldsymbol{p}-\boldsymbol{q})\right]\left(\boldsymbol{p}, \boldsymbol{q}\left|\mathscr{W}_{1}(\chi)\right| \boldsymbol{p}^{\prime}, \boldsymbol{q}^{\prime}\right) \\
& \times \exp \left[-\mathrm{i} \boldsymbol{R}_{1} \cdot\left(\boldsymbol{p}^{\prime}-\boldsymbol{q}^{\prime}\right)\right] .
\end{aligned}
$$

This implies that the $R$-integral for each of the terms of the binary collision expansion simply yields a delta function. The two-collision term, $\mathscr{W}_{1}\left(\frac{1}{2} \boldsymbol{R}\right) \mathscr{G}_{0} \mathscr{W}_{1}\left(-\frac{1}{2} \boldsymbol{R}\right)$, is readily found to reduce to the $\mathcal{O}\left(\epsilon^{-3}\right)$ term of $\hat{\boldsymbol{S}}_{2}(\epsilon)$. For 
the triple collision term we get

$$
\begin{aligned}
\hat{S}_{2}^{3}(\epsilon)= & 2 \mathrm{i} \epsilon^{-2}(2 \pi)^{d} \int \mathrm{d} p \mathrm{~d} p^{\prime} \mathrm{e}^{-\beta p^{2}}\left(p \cdot p^{\prime}\right) \int \mathrm{d} p_{1} \mathrm{~d} q_{1} \mathrm{~d} p_{2} \mathrm{~d} q_{2} \delta\left(p_{1}-q_{1}-p_{2}+q_{2}\right) \\
& \times \frac{\left(p, p\left|W_{1}(\chi)\right| p_{1}, q_{1}\right)\left(p_{1}, q_{1}\left|W_{1}(\chi)\right| p_{2}, q_{2}\right)\left(p_{2}, q_{2}\left|W_{1}(\chi)\right| p^{\prime}, p^{\prime}\right)}{\left[\chi-\left(p_{1}^{2}-q_{1}^{2}\right)\right]\left[\chi-\left(p_{2}^{2}-q_{2}^{2}\right)\right]}
\end{aligned}
$$

This expression is strictly analogous to the triple collision contribution in a real quantum gas as found by Albers and Oppenheim ${ }^{8}$ ). These authors also discussed that if one puts $p_{1}-q_{1}=p_{2}-q_{2}=0$ in the numerator this is just the classical expression which is known to exhibit a $\log \epsilon$ in two dimensions. Carrying out this analysis in eq. (4.16) we found the same logarithmic term as we did in eq. (3.21). Retaining only the diagonal matrix elements in the numerator of eq. (4.16) means that the off-shell effects are ignored. The numerical analysis of the full two-scatterer contribution, that we mentioned in the previous section confirms that the off-shell structure of the numerator does not influence the $\log \epsilon$ behaviour.

Because of the strict analogy of the triple collision contribution for the quantum Lorentz gas and the real quantum gas, there seems to be no reason to doubt the validity of the existing $\log \epsilon$ estimates for both systems. $\mathrm{Eu}^{15}$ ) asserts that the Albers and Oppenheim analysis is incorrect and claims that there is no $\log \epsilon$ problem for real gas. These assertions are based on algebraic errors and consequently wrong.

\section{Boundstate contributions to the velocity autocorrelation function}

In this section we will consider a quantum Lorentz gas with attractive interactions between the light particle and the scatterers in order to study the influence of boundstates on $\hat{\boldsymbol{S}}(\epsilon)$. In ref. 10 the boundstate contributions to the first two cluster functions have been studied directly by extracting the corresponding part out of the traces in eqs. (2.8)-(2.9). Here we will discuss this problem starting from the Laplace transformed $T$-matrix expressions for $\hat{S}_{1}$ and $\hat{S}_{2}(2.21)-(2.26)$. In this way we can interpret the results in terms of the analytic structure of the $T$-matrix elements. The pure boundstate contributions can be found from the real collision terms, this will be domonstrated for $\hat{S}_{2}(\epsilon)$.

Our separable potential is chosen to be sufficiently attractive (i.e. $l \equiv$ $2 \pi^{2} \alpha^{-1} \lambda<-1$ ) to have a one scatterer boundstate $\psi_{0}$ with an energy $\epsilon_{0}$. As discussed in $I$ this implies that if $R$ is smaller than some critical value $\boldsymbol{R}_{0}$, there is precisely one way the light particle can be bound to two scatterers; the corresponding wave function $\psi_{+}$being even. If $R \geqslant R_{0}$ there is also an odd 
boundstate $\psi_{-}$. Its energy $\epsilon_{-}$vanishes in the limit $R \downarrow R_{0}$, but for a threedimensional system it is still a proper boundstate in the sense that $\psi_{\text {-remains }}$ normalisable. Some details about the boundstate properties are given in appendix $B$. The resulting analytic structure of the $\hat{S}_{2}^{\mathrm{r}}$-integrand (2.24) in the complex $z$-plane is shown in fig. 3 for the case that $R>R_{0}$ and $\epsilon=\eta+i \omega$. The Watson contour $C$ (2.10)-(2.11) has been divided into three pieces in order to select out the various physical processes. For our present purpose it is convenient to rewrite $\hat{S}_{2}^{\mathrm{r}}(\epsilon)$ in terms of resolvents using eq. (2.12),

$$
\begin{aligned}
\hat{S}_{2}^{\mathrm{r}}(\boldsymbol{\epsilon})= & -(2 \pi)^{-1} \int \mathrm{d} \boldsymbol{R} \int \mathrm{d} \boldsymbol{p} \mathrm{d} p^{\prime}\left(\boldsymbol{p} \cdot \boldsymbol{p}^{\prime}\right) \oint \mathrm{d} z \mathrm{e}^{-\beta z} \\
& \times\left\langle\boldsymbol{p}\left|G_{2}(z-\mathrm{i} \epsilon)-G_{0}(z-\mathrm{i} \epsilon)\right| \boldsymbol{p}^{\prime}\right\rangle\left\langle\boldsymbol{p}^{\prime}\left|G_{2}(z)-G_{0}(z)\right| p\right\rangle .
\end{aligned}
$$

The integrations along $C_{+}$and $C_{-}$can be done simply with the use of the following spectral decomposition of the two-scatterer Greens function

$$
G_{2}(z)=\frac{\left|\psi_{+}\right\rangle\left\langle\psi_{+}\right|}{z-\epsilon_{+}}+\Theta\left(R-R_{0}\right) \frac{\left|\psi_{-}\right\rangle\left\langle\psi_{-}\right|}{z-\epsilon_{-}}+\int_{0}^{\infty} \mathrm{d} E_{2} \frac{\left|E_{2}\right\rangle\left\langle E_{2}\right|}{z-E_{2}},
$$

where $\left|E_{2}\right\rangle$ are the scattering eigenstates of $H_{2}$. Retaining only the pole terms of the left Greens function we obtain the pure boundstate contribution, $\hat{S}_{2}^{b}$, to $\hat{S}_{2}$,

$$
\hat{S}_{2}^{b}(\epsilon)=-\mathrm{i} \int_{R \geqslant R_{0}} \mathrm{~d} \mathbf{R}\left|\left\langle\psi_{+}|\boldsymbol{P}| \psi_{-}\right\rangle\right|^{2}\left[\frac{\exp \left(-\beta \epsilon_{+}\right)}{\epsilon_{+}-\epsilon_{-}-\mathrm{i} \epsilon}-\frac{\exp \left(-\beta \epsilon_{-}\right)}{\epsilon_{+}-\epsilon_{-}+\mathrm{i} \epsilon}\right] .
$$

We note that two out of the four possible terms are zero because $\left\langle\psi_{ \pm}|\boldsymbol{P}| \psi_{ \pm}\right\rangle=$ 0 . For the same reason there is no pure boundstate contribution to the one-scatterer cluster functions.

Let us consider $\hat{S}_{2}(\eta+i \omega)$ in the limiting case that $\eta \downarrow 0$. Looking at fig. 3 we see that in this limit and for $0<\omega \leqslant \omega_{0} \equiv-\epsilon_{+}\left(R_{0}\right)$ there exists an $R \geqslant R_{0}$ such that the $C_{+}$contour will be pinched between the even boundstate pole

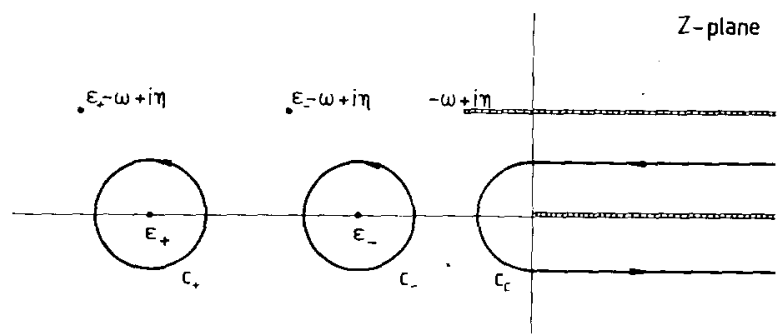

Fig. 3. Singularity structure of the $\hat{S}_{2}^{r}$-integrand $\left(l<-1, R>R_{0}\right)$. 
and the shifted odd boundstate pole, similarly $C_{-}$can be pinched between $\epsilon_{-}$ and $\epsilon_{+}+i \epsilon$ if $-\omega_{0} \leqslant \omega<0$. The pinches will show up as a divergence at the real $R$-axis in the integrand of eq. (5.3). As long as these singular $R$-values are larger than $R_{0}$, i.e. $|\omega|<\omega_{0}$, it is possible to deform the $R$-contour away from the singularity and the resulting contribution to $\hat{S}_{2}(i \omega)$ will be finite. However if $\omega=\omega_{0}$, the pinch will occur if $R=R_{0}$, in which case the odd poles are about to leave to physical $z$-plane. This causes and end-point singularity in the $R$-integrand of eq. (5.3) and a logarithmic divergence of $\hat{S}_{2}(\mathrm{i} \omega)$ if $\omega \rightarrow \omega_{0}$; provided that the residue at the pinching pole does not vanish. As a result the frequency dependent electrical conductivity will exhibit a logarithmic divergence.

If we suppose the light particle to be an electron with charge $e$ and mass $m$, linear response theory ${ }^{16}$ ) leads to the following expression for the conductivity tensor:

$$
\sigma_{a b}(\omega)=-\left(i e^{2} / \omega m\right) \delta_{a b}+\left(e^{2} / \hbar \omega m^{2}\right) \int_{0}^{\infty} \mathrm{d} t \mathrm{e}^{-i \omega t}\left\langle\left[P_{b}(0), P_{a}(t)\right]\right\rangle .
$$

Hence for an isotropic system the relation between the correlation function $\hat{S}$ and the frequency dependent conductivity $\sigma(\omega)$ is the following,

$$
\begin{aligned}
& \sigma(\omega)=-(2 \mathrm{i} / \omega)+(8 / \omega) \tilde{\sigma}(\omega), \\
& \tilde{\sigma}(\omega)=(2 d)^{-1} \lim _{\eta \downarrow 0}\left[\hat{S}(\epsilon)-\hat{S}^{*}\left(\epsilon^{*}\right)\right]
\end{aligned}
$$

(again we have adapted units in which $-e=2 m=\hbar=1$ ). If we combine eq. (5.3) and eq. (5.6) and introduce an integration variable $E \equiv \epsilon_{-}-\epsilon_{+}$instead of $R$, we get for the two-scatterer boundstate contribution to $\tilde{\sigma}(\omega)$

$$
\begin{aligned}
\tilde{\sigma}_{2}^{\mathrm{b}}(\omega)= & -\mathrm{i} \pi(d-1) d^{-1} \lim _{\eta \downarrow} \int_{0}^{\omega_{0}} \mathrm{~d} E\left[\frac{\mathrm{d} R}{\mathrm{~d} E} R^{d-1}\left|\left\langle\psi_{+}|P| \psi_{-}\right\rangle\right|^{2}\left(\mathrm{e}^{-\beta \epsilon_{+}}-\mathrm{e}^{-\beta \epsilon_{-}}\right)\right. \\
& \left.\times\left(\frac{1}{E-\omega+\mathrm{i} \eta}+\frac{1}{E+\omega-\mathrm{i} \eta}\right)\right] .
\end{aligned}
$$

From this equation we see that $\tilde{\sigma}_{2}^{b}(\omega)$ is an analytic function of $\omega$ with a cut from $-\omega_{0}$ to $\omega_{0}$. As discussed in appendix B the normalisability of $\psi_{-}$at $R=R_{0}$ implies that the overlap matrix element does not vanish in three dimensions if $E \rightarrow \omega_{0}$. Moreover, since $R^{\prime}\left(\omega_{0}\right)<0$ we see that the residues at the pinching poles do not go to zero so that $\tilde{\sigma}_{2}^{\mathrm{b}}(\omega)$ has a logarithmic singularity at $\omega= \pm \omega_{0}$. In fig. 4 is shown the frequency dependence of the real and imaginary part of $\tilde{\sigma}_{2}^{\mathrm{b}}(\omega)$ for a three-dimensional system with separable 

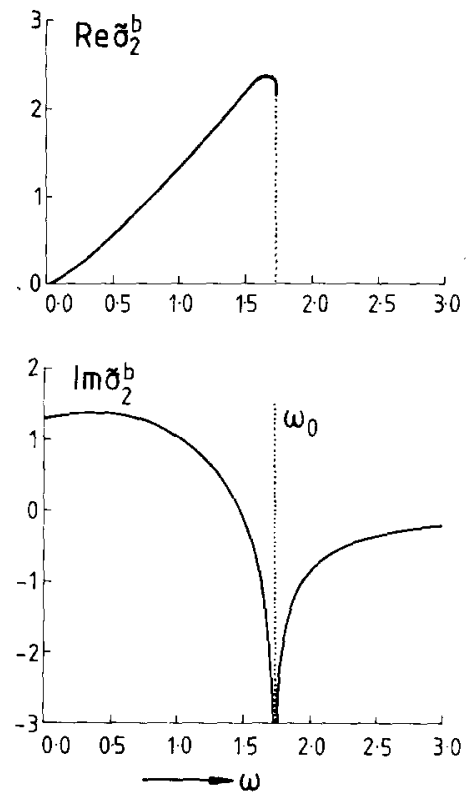

Fig. 4. The three-dimensional conductivity plotted as a function of the frequency of the electric field for a sufficiently attractive potential $(l=-2, \alpha=1)$ at some arbitrary temperature $(\beta=1)$. $\operatorname{Re} \tilde{\sigma}_{2}^{\mathrm{b}}$ drops to zero discontinuously if $\omega=\omega_{0}$.

effective range interactions. From this we see that near $\omega=\omega_{0} \operatorname{Im} \tilde{\sigma}_{2}^{b}$ diverges, while $\operatorname{Re} \tilde{\sigma}_{2}^{\mathrm{b}}$ vanishes for $\omega>\omega_{0}$.

Regarding the singularity structure of the $\hat{S}_{2}(\epsilon)$-integrand, as shown in fig. 3 , it is clear that no other pinches wil occur simultaneously. This implies that the divergence will not be compensated by continuum contributions. We already mentioned that there is no pure boundstate contribution to $\hat{S}_{1}(\epsilon)$. For the higher cluster functions $\hat{S}_{n}(\epsilon), n \geqslant 3$, essentially the same singularity structure in the $z$-plane is found as in fig. 3. There will be pinches as well. However, instead of carrying out a one-dimensional integral to average over all scatterer positions, we must in those cases integrate in at least a three-dimensional configuration space, in which the pinches show up as a singular hyper surface which is of logarithmic type so the integration yields a finite result. This indicates that the divergence in $\tilde{\sigma}_{2}(\omega)$ will also be present in the complete conductivity $\sigma(\omega)$.

The singular behaviour of $\hat{S}_{2}(\epsilon)$ is a signal of a slowly decaying long time tail of the VACF in three dimensions which can readily be found by carrying out the inverse Laplace transformation of $\hat{S}_{2}^{b}(\epsilon)$, eq. (5.3),

$$
\langle\boldsymbol{P} \cdot \boldsymbol{P}(t)\rangle \approx 2 \pi \mathrm{i} \rho^{2} \Lambda^{3}\left\{\frac{\mathrm{d} R}{\mathrm{~d} E}\left|\left\langle\psi_{+}|\boldsymbol{P}| \psi_{-}\right\rangle\right|^{2}\right\}_{R=R_{0}} \boldsymbol{R}_{0}^{2} \mathrm{t}^{-1}\left\{\mathrm{e}^{\beta \omega_{0}} \mathrm{e}^{\mathrm{i} \omega_{0} t}-\mathrm{e}^{-\mathrm{i} \omega_{0} t}\right\} .
$$


As discussed in appendix $B$, in two dimensions the overlap matrix element vanishes in such a way that $\left|\left\langle\psi_{+}|\boldsymbol{P}| \psi_{-}\right\rangle\right|^{2} \sim\left|\log \left(R-R_{0}\right)\right|^{-1}$. As a consequence $\tilde{\sigma}_{2}^{b}(\omega)$ behaves like $\log |\log | \omega-\omega_{0}||$ in that case. In one dimension the normalisation constant vanishes in a regular way near $R=R_{0}$ so that no divergence is present in $\sigma(\omega)$.

\section{Conclusions}

We have derived a contour integral expression for the coefficients of the density expansion of the Laplace transformed VACF, which can be considered as an extension of Fano's formula in order to include the statistical correlation effects. The assumed separability of the interaction enabled us to give explicit expressions for the off-shell $T$-matrix elements that occur in these coefficients. We gave a detailed demonstration of the logarithmic divergence of the two-scatterer contribution to the coefficient of self-diffusion in two dimensions. Numerically we have evaluated the complete two-scatterer contribution to $\hat{S}(\epsilon)$ over a wide range of $\epsilon$-values, including the exact off-shell $T$-matrix elements and the statistical correlation effects. The results of these calculations for small values of $\epsilon$ agree with the usual simple estimates for the singular terms. Hence we conclude that quantum mechanical off-shell effects do not change the established classical behaviour of the diffusion coefficient. The analysis of the diffusion problem in terms of Liouville operators showed that the triple collision term in both the quantum Lorentz gas and the real quantum gas are strictly analogous, hence there seems to be no reason to doubt the results of Albers and Oppenheim ${ }^{8}$ ).

Finally we considered the influence of boundstate processes on the VACF. We have shown that for our simple model the boundstate part of the two-scatterer contribution in three dimensions exhibits a long time tail, which leads to a logarithmic divergence in the frequency dependent electrical conductivity. The physical prerequisite for the phenomenon to occur is the existence of a two-scatterer boundstate which is shifted into the continuum if the scatterers are being put closer together with the property that it remains normalisable at the threshold. As we mentioned in appendix $B$ the odd zero-energy states of a local finite range potential are also normalisable, hence it is expected that the condition is also satisfied for a local potential that exclusively admits one $1=0$ boundstate of the light particle with one scatterer and with two scatterers put at the same position. Since the quantum Lorentz gas may serve as a simple model for conduction of electrons in a disordered system when the interaction between them can be neglected, a measurement of the frequency dependent conductivity of such a system would be interes- 
ting to see the existence of this slowly decaying tail in the velocity autocorrelation function.

\section{Appendix A}

\section{Some asymptotic relations}

In this appendix we give details on the derivation of the long distance behaviour of the various functions needed in section 3 . For the $S$-wave effective range potential the two-dimensional denominator functions (3.4)-(3.5) are

$$
\begin{aligned}
& \Delta(z)=1-\left[\pi \lambda /\left(z+\alpha^{2}\right)\right] \log \left(-z / \alpha^{2}\right), \\
& D(R ; z)=-\left[2 \pi \lambda /\left(z+\alpha^{2}\right)\right]\left[K_{0}(R \sqrt{-z})-K_{0}(R \alpha)\right],
\end{aligned}
$$

where $K_{0}$ is the zeroth order modified Bessel function. These functions have the following asymptotic form ${ }^{13}$ )

$$
K_{n}(w)=\sqrt{\frac{\pi}{2 w}} \mathrm{e}^{-w}\left[1+\frac{4 n^{2}-1}{8 w}+\mathcal{O}\left(w^{-2}\right)\right] .
$$

Combination of eq. (A.2) and eq. (A.3) immediately yields the asymptotic form of $D$, eq. (3.10).

For our potential the two-dimensional trace integrals, eq. (3.15), can also be done analytically. The angular integrations give rise to first order Bessel functions

$$
\begin{aligned}
& \int \mathrm{d} \hat{\boldsymbol{p}} \mathrm{d} \hat{p}^{\prime}\left(\hat{\boldsymbol{p}} \cdot \hat{\boldsymbol{p}}^{\prime}\right) \cos ^{2} \frac{1}{2} \mathbf{R} \cdot\left(\boldsymbol{p}+\boldsymbol{p}^{\prime}\right)=-2 \pi^{2} J_{1}(R p) J_{1}\left(R p^{\prime}\right), \\
& \hat{\boldsymbol{p}}=\boldsymbol{p} /|\boldsymbol{p}|,
\end{aligned}
$$

so that

$$
\begin{aligned}
\tau(R ; z, \epsilon)= & -2 \pi^{2} \epsilon^{2}\left[\int_{0}^{\infty} \mathrm{d} p \frac{p^{2} g^{2}(p) J_{1}(R p)}{\left(z-p^{2}-\mathrm{i} \epsilon\right)\left(z-p^{2}\right)}\right]^{2} \\
= & 2 \pi^{2}\left[\left(z+\alpha^{2}-\mathrm{i} \epsilon\right)^{-1}\left\{\sqrt{-z+\mathrm{i} \epsilon} K_{1}(R \sqrt{-z+\mathrm{i} \epsilon})-\alpha K_{1}(R \alpha)\right\}\right. \\
& \left.-\left(z+\alpha^{2}\right)^{-1}\left\{\sqrt{-z} K_{1}(R \sqrt{-z})-\alpha K_{1}(R \alpha)\right\}\right] .
\end{aligned}
$$

With the use of the asymptotic expansion of $K_{1}$, eq. (A.3), we find from this expression the asymptotic form of the two-dimensional trace integrals, eq. (3.18).

Next we consider the three-dimensional case. The denominator functions 
are given by

$$
\begin{aligned}
& \Delta(z)=1+2 \pi^{2} \lambda /(\alpha+\sqrt{-z}), \\
& D(R ; z)=-\left(\frac{2 \pi^{2} \lambda}{R}\right)\left[\frac{\exp (-R \sqrt{-z})-\exp (-R \alpha)}{\left(z+\alpha^{2}\right)}\right] .
\end{aligned}
$$

The multiple scattering series for the virtual two-scatterer contribution can be obtained from its two-dimensional counterpart, eq. (3.9), by replacing the zeroth order Bessel function $J_{0}(R p)$ by $(R p)^{-1}$ sin $R p$. The integral expression for $\hat{S}_{2}^{\vee 2}$, eq. (3.8), gets an extra factor $R$ in the numerator. For $\epsilon \downarrow 0$ none of the exponentials of the odd part (i.e. the first term) of eq. (3.9) goes one so that a finite contribution to $\epsilon^{2} \hat{S}_{2}^{\mathrm{v} 2}$ results. In the even contribution we see that in each term the oscillations of the sine and the denominator functions can extinguish each other. Counting the factors $R^{-1}$ we see that the $n_{+}=0$ term gives rise to the $\mathscr{O}\left(\epsilon^{-3}\right)$ contribution to $\hat{S}_{2}^{\mathrm{v} 2}$. All other terms are damped at least proportional to $R^{-2}$, hence giving a finite contribution to $\epsilon^{2} \hat{S}_{2}^{v 2}$.

Subsequently we regard the three-dimensional real two-scatterer terms. The angular trace integrals, eq. (3.15), can be carried out readily,

$$
\int \mathrm{d} \hat{\boldsymbol{p}} \mathrm{d} \hat{\boldsymbol{p}}^{\prime}\left(\hat{\boldsymbol{p}} \cdot \hat{\boldsymbol{p}}^{\prime}\right) \cos ^{2} \frac{1}{2} \boldsymbol{R} \cdot\left(\boldsymbol{p}+\boldsymbol{p}^{\prime}\right)=-8 \pi^{2} B_{+}(R p) B_{+}\left(R p^{\prime}\right),
$$

where

$$
\begin{aligned}
& B_{ \pm}(w)=b^{+}(w) \pm b^{-}( \pm w), \\
& b^{+}(w)=-\frac{1}{2} \mathrm{e}^{i w}\left(\frac{\mathrm{i}}{w^{2}}+\frac{1}{w}\right), \\
& b^{-}(w)=-\frac{1}{2} \mathrm{e}^{-i w}\left(-\frac{\mathrm{i}}{w^{2}}+\frac{1}{w}\right)
\end{aligned}
$$

Some straightforward algebra yields for the complete trace integrals

$$
\begin{aligned}
\tau(R ; z, \epsilon)= & -2 \pi^{3}\left[-\left(\frac{z-\mathrm{i} \epsilon}{z+\alpha^{2}-\mathrm{i} \epsilon}\right) B_{-}(\mathrm{i} R \sqrt{-z+\mathrm{i} \epsilon})+\left(\frac{z}{z+\alpha^{2}}\right) B_{-}(\mathrm{i} R \sqrt{-z})\right. \\
& \left.-\left(\frac{\alpha^{2}}{z+\alpha^{2}-\mathrm{i} \epsilon}\right) B_{-}(\mathrm{i} \alpha R)+\left(\frac{\alpha^{2}}{z+\alpha^{2}}\right) B_{-}(\mathrm{i} \alpha R)\right]^{2}
\end{aligned}
$$

Working this out for $z=E+\frac{l}{2} \mathrm{i} \epsilon$ and small $\epsilon$ one finds

$$
\begin{aligned}
\tau\left(R ; E+\frac{1}{2} \mathrm{i} \epsilon, \epsilon\right)= & -\left(2 \pi^{3} E^{2} g^{4}(p) / R^{2} E\right)\left[\exp \left(-2 R \sqrt{-E+\frac{1}{2} i \epsilon}\right)\right. \\
& \times\left(1-\frac{2 \mathrm{i}}{R p}-\frac{1}{R^{2} E}\right)+\exp \left(-2 R \sqrt{-E-\frac{1}{2} \mathrm{i} \epsilon}\right)
\end{aligned}
$$




$$
\begin{aligned}
& \times\left(1+\frac{2 \mathrm{i}}{R p}-\frac{1}{R^{2} E}\right)+2 \exp \left(-R\left[\sqrt{-E+\frac{1}{2} \mathrm{i} \epsilon}+\sqrt{-E-\frac{1}{2} \mathrm{i} \epsilon}\right]\right) \\
& \left.\times\left(1+\frac{1}{R^{2} E}\right)\right]+\mathcal{O}\left(R^{-2} \mathrm{e}^{-R \alpha}\right) .
\end{aligned}
$$

If we compare this expression with eq. (3.18) we see that $R^{d-1} \times \tau$ is essentially of the same form, irrespective whether $d=2$ or $d=3$. Now we can find the asymptotic behaviour of $\hat{S}_{2}^{r}$ at once by looking at the multiple scattering series, eq. (3.17). The term with $n_{-}=m_{-}=0$ together with the third term of the trace integral, eq. (A.13), gives a Zwanzig term plus a regular contribution. All other terms in the multiple scattering series fall off at least as rapid as $R^{-2}$ since $|D| \sim R^{-1}$. This implies that the three-dimensional two-scatterer contribution to the coefficient of self-diffusion is finite.

\section{Appendix B}

\section{Two-scatterer boundstate properties}

In this appendix we discuss the properties of the two-scatterer boundstate wave function for the case of separable interactions and also for the more familiar case of local finite range interactions.

For the separable case the required information is to be found from the following Schrödinger equation,

$$
\left[H_{0}+\lambda\left|g_{+}\right\rangle\left\langle g_{+}|+\lambda| g_{-}\right\rangle\left\langle g_{-}\right|\right]|\psi\rangle=\epsilon_{b}|\psi\rangle,
$$

where

$$
\left|g_{ \pm}\right\rangle \equiv \exp \left( \pm \frac{1}{2} \mathrm{iR} \cdot \boldsymbol{P}\right)|g\rangle .
$$

Hence the wave function is of the following form:

$$
|\psi\rangle=\alpha_{+}\left(\epsilon_{\mathrm{b}}-H_{0}\right)^{-1}\left|g_{+}\right\rangle+\alpha_{-}\left(\epsilon_{\mathrm{b}}-H_{0}\right)^{-1}\left|g_{-}\right\rangle,
$$

which leads to consistency relations

$$
\begin{aligned}
& {\left[1-\lambda\left\langle g_{+}\left|\left(\epsilon_{\mathrm{b}}-H_{0}\right)^{-1}\right| g_{+}\right\rangle\right] \alpha_{+}-\lambda\left\langle g_{+}\left|\left(\epsilon_{\mathrm{b}}-H_{0}\right)^{-1}\right| g_{-}\right\rangle \alpha_{-}=0,} \\
& -\lambda\left\langle g_{-}\left|\left(\epsilon_{\mathrm{b}}-H_{0}\right)^{-1}\right| g_{+}\right\rangle \alpha_{+}+\left[1-\lambda\left\langle g_{-}\left|\left(\epsilon_{\mathrm{b}}-H_{0}\right)^{-1}\right| g_{-}\right\rangle\right] \alpha_{-}=0,
\end{aligned}
$$

which allow a nontrivial solution only if the energy has one of the two values $\epsilon_{ \pm}$that satisfy (cf. eqs. (3.4)-(3.5))

$$
\Delta\left(\epsilon_{ \pm}\right)= \pm D\left(R ; \epsilon_{ \pm}\right),
$$

so that we find two possible boundstate wave functions

$$
\psi_{ \pm}(p)=\mathcal{N}_{ \pm} g(p)\left(\epsilon_{ \pm}-p^{2}\right)^{-1}\left[\exp \left(\frac{1}{2} \mathrm{i} R \cdot p\right) \pm \exp \left(-\frac{1}{2} \mathrm{i} R \cdot \boldsymbol{p}\right)\right] .
$$


The normalisation constants are given by

$$
\mathcal{N}_{ \pm}^{-2}(R)=2 \int \mathrm{dpg}^{2}(p)\left(\epsilon_{ \pm}-p^{2}\right)^{-2}(1 \pm \exp (\mathrm{iR} \cdot p))
$$

For the odd case it is readily seen that both in two and three dimensions the angular integral of the $R$-dependent factor becomes $O\left(p^{2}\right)$ for small $p$, so that in the limit $\epsilon_{-} \uparrow 0 \mathcal{N}_{-}$remains finite if $d=3$ while $\mathcal{N}_{-}^{-2}=\mathscr{O}\left(\log \left(R-R_{0}\right)\right)$ in two dimensions. The overlap matrix element $\left|\left\langle\psi_{+}|\boldsymbol{P}| \psi_{-}\right\rangle\right|$behaves similar to $\mathcal{N}_{-}$ since the extra integral one has to evaluate in that case is regular in the limit $\epsilon_{-} \uparrow 0$.

The normalisability of an odd zero energy wave function is not just a peculiarity of the separable interaction. If the interaction is of finite range $r_{0}$ the zero energy wave function $\psi_{0}(r)$ has to satisfy the Laplace equation if $|r|>\frac{1}{2} R+r_{0}$. The odd solutions of this equation which go to zero at infinity are such that

$$
\psi_{0}^{-}(r)=\mathscr{O}\left(r^{-(d-1)}\right) \quad(d=2,3) .
$$

Hence we see that for this case true zero energy boundstates can exist in three dimensions as well, and that again the normalisation constant diverges logarithmically in two dimensions.

\section{References}

1) For a recent review see J.R. Dorfman, Physica 106A (1981) 77.

2) K. Kawasaki and I. Oppenheim, Phys. Rev. 139A (1965) 1763; Statistical Mechanics, T.A. Bak ed. (Benjamin, New York, 1967); Y. Kan and J.R. Dorfman, Phys. Rev. 16A (1977) 2447.

3) B.J. Alder and T.E. Wainwright, Phys. Rev. Lett. 18 (1967) 988; Phys. Rev. 1A (1970) 18.

4) J.R. Dorfman and E.G.D. Cohen, Phys. Rev. 6A (1972) 776; 12A (1975) 292.

5) J.M.J. van Leeuwen and A. Weijland, Physica 36A (1967) 457; A. Weijland and J.M.J. van Leeuwen, Physica 38A (1968) 35.

6) E.H. Hauge, in Lecture Notes in Physics, vol. 31, G. Kirczenov and J. Marro, eds. (Springer, Berlin, 1974); H. van Beijeren, Rev. Mod. Phys. 54 (1982) 195.

7) M.H. Ernst and A. Weijland, Phys. Lett. 34A (1971) 39; C. Bruin, Phys. Rev. Lett. 29 (1972) 1670, Physica 72 (1974) 261; J.C. Lewis and J.A. Tjon, Phys. Lett. 66A (1978) 349; B.J. Alder, in Lecture Notes in Physics, vol. 84, L. Garrido, P. Seglar and P.J. Shepard, eds. (Springer, Berlin, 1978); B.J. Alder and W.E. Alley, J. Stat. Phys. 19 (1978) 314; W.E. Alley and B.J. Alder, Phys. Rev. Lett. 43 (1979) 653,

8) J. Albers and I. Oppenheim, Physica 59 (1972) 161, 187.

9) P. Résibois and M.G. Velarde, Physica 51 (1971) 541.

10) W. Hoogeveen and J.A. Tjon, Phys. Lett. 86A (1981) 353.

11) W. Hoogeveen and J.A. Tjon, Physica 108A (1981) 77, to be referred to as I.

12) R. Zwanzig, Phys. Rev. 129 (1963) 486.

13) M. Abramowitz and I.A. Stegun, Handbook of Mathematical Functions (Dover, New York, 1972). 
I.S. Gradshteyn and I.M. Ryzhik, Tables of Integrals Series and Products (Academic Press, New York, 1965).

14) U. Fano, Phys. Rev. 131 (1963) 259.

15) B.C. Eu, J. Chem. Phys. 60 (1974) 1906, the crucial error is made in eq. (3.26) of this paper: the incomplete elliptic integral $F\left(\pi / 4, \epsilon^{1 / 2}(1+\epsilon)^{-1 / 2}\right)$ which yields a finite bound has to be replaced by a complete elliptic integral $F\left(\pi / 2,(1+\epsilon)^{-1 / 2}\right)$ which is $O(\log \epsilon)$ for $\epsilon \rightarrow 0$; J. Chem. Phys. 60 (1974) 1914, section $V$ of this paper contains several errors which lead to the wrong conclusion (eq. (5.19)) that the complete three-body contribution to the density expansion of the diffusion coefficient is $O\left(\epsilon^{-2}\right)$ while it is well-known to be $O\left(\epsilon^{-3}\right)$.

16) R. Kubo, Can. J. Phys. 34 (1956) 1274; J. Phys. Soc. Japan 12 (1957) 570; Rep. Prog. Phys. 29 (1966) 255; E. Verboven, Physica 26 (1960) 1091. 